VIH

et protéines $G$

Le premier semestre 1996 est exceptionnel en ce qui concerne les connaissances sur la biologie du VIH et les perspectives thérapeutiques du SIDA. L'élément pronostique le plus important dans cette maladie est la charge virale et son évolution, d'où les espoirs engendrés par les multithérapies modernes, incluant des antiprotéases, qui peuvent aboutir à une quasi-disparition du virus détectable chez les malades. La primo-infection par le VIH nécessite que les cellules cibles aient d'autres déterminants que le récepteur $\mathrm{CD} 4$. Ceux-ci viennent d'être identifiés : ce sont des récepteurs de chimiokines (RANTES, MIP $\alpha$ et MIP $\beta$ ), ou de facteurs encore non identifiés, molécules à sept passages transmembranaires et couplées aux protéines $G$. Ces résultats expliquent l'effet antiviral de chimiokines, récemment rapporté : ces facteurs entreraient en compétition avec le virus pour la liaison aux récepteurs dont ils induiraient la régulation négative par endocytose. L'importance de ces récepteurs est attestée par la résistance à l'infection virale des sujets dont le gène correspondant est muté. Les protéines $G$ pourraient être impliquées dans d'autres phénomènes où on ne les attendait pas, par exemple la maladie d'Alzheimer. En effet, la protéine précurseur du peptide $\beta$-amyloïde pourrait être un récepteur couplé à une protéine Go. En revanche, le récepteur de la thrombine, précédemment caractérisé, couplé lui aussi à une protéine $G$, n'explique en rien les effets de cette protéase sur la coagulation sanguine.

\title{
Les facteurs cellulaires de fusion avec VIH enfin identifiés
}

L'identification par l'équipe d'Edward Berger (NIH, Bethesda, MD, USA) d'un facteur cellulaire permettant l'entrée du virus de l'immunodéficience humaine (VIH-1) dans les cellules [1], est l'aboutissement d'une quête menée depuis dix ans, et une avancée scientifique considérable dans le domaine. Ce premier facteur permet l'entrée d'une certaine catégorie de souches de VIH-1, dites «de laboratoire » ou «adaptées» (aux lignées cellulaires immortalisées), qui diffèrent par de nombreuses propriétés des souches "primaires» et/ou «macrophage-tropiques", responsables de l'établissement et de la persistance de l'infection chez les patients. Mais sa découverte a rapidement permis au même groupe [2] et à deux autres équipes [3,4] d'identifier un autre facteur cellulaire permettant l'entrée des souches primaires.

On sait depuis 1984 le rôle que joue CD4 dans l'entrée de VIH-1, et cette molécule est d'ailleurs le premier récepteur rétroviral identifié $(\mathrm{m} / \mathrm{s}$ $n^{\circ} 3$, vol. 3, p. 180). Dès qu'un clone moléculaire fut disponible, Maddon et al. [5] montrèrent en 1986 que l'expression de CD4 suffisait à rendre les cellules humaines permissives à l'infection par VIH-1. Les mêmes auteurs observèrent que les cellules murines $\mathrm{CD}^{+}$fixaient le virus sans s'infecter, ni pouvoir fusionner avec des cellules exprimant les glycoprotéines d'enveloppe de VIH-1 (Env, gp120/gp41). Cela suggérait que seules les cellules humaines possédaient un facteur nécessaire à cette étape de fusion membranaire, parfois appelé «deuxième récepteur " de VIH-1. Beaucoup d'énergie fut dépensée au cours des dix années suivantes pour rechercher ce ou ces facteurs de fusion, et le rôle d'un nombre considérable de molécules de surface fut testé sans pouvoir obtenir de preuve directe. On ne revien- dra pas sur le cas de CD26, affirmé avec une médiatisation extrême être le cofacteur d'entrée de VIH-1 $(\mathrm{m} / \mathrm{s}$ $n^{\circ} 1$, vol. 10, p. 117). Ce résultat reposait sur des expériences techniquement discutables, et ne put être confirmé par des tests directs [6].

Toutes les tentatives de complémentation de cellules animales avec du matériel génétique humain (ADN génomique ou banques d'ADNc) échouèrent dans différents laboratoires. Par ailleurs, les hybrides somatiques souris-homme $\mathrm{CD} 4^{+}$sont résistants à VIH-1, quel que soit leur contenu en chromosomes humains [7]. On pouvait donc se demander si les cellules animales n'exprimaient pas des facteurs empêchant l'entrée de VIH, rendant toute complémentation impossible. En testant des hybrides cellulaires souris-homme immédiatement après leur formation, c'est-à-dire sous forme d'hétérokaryons, nous avons montré que la complémentation était possible, et donc confirmé l'existence des facteurs de fusion [8]. Nous avons observé leur présence dans différentes lignées cellulaires humaines, et dans les globules rouges où ils sont associés à la membrane plasmique et apparemment résistants aux traitements protéolytiques [9]. Les deux types de VIH utiliseraient des facteurs de fusion différents, car certaines lignées sont permissives à VIH-2 et non à VIH-1 [10].

La réussite de l'équipe de Berger, là où tant d'autres avaient échoué, tient à l'emploi d'une technologie de complémentation génétique différente, et certainement plus puissante. Il s'agit du système d'expression constitué du couple promoteur T7/ARN polymérase du bactériophage T7 (T7pol), classiquement utilisé pour la transcription d'ARN in vitro. En transfectant des cellules avec un gène placé sous contrôle du promoteur 
T7, puis en les infectant par un virus vaccine recombinant exprimant $T 7 p o l$, on peut exprimer ce gène à un niveau très élevé. En effet, les hauts titres infectieux du virus vaccine permettent de toucher toutes les cellules transfectées, et d'y synthétiser de grandes quantités de T7pol. Les ARN $T 7$, produits dans le cytoplasme, n'ont pas la coiffe 5' nécessaire à leur traduction, mais la machinerie du virus vaccine permet la modification d'une fraction suffisante de ces transcrits pour produire 10 à 100 fois plus de protéines qu'avec les systèmes d'expression usuels.

Feng et al. [1] ont donc co-transfecté des cellules murines (lignée NIH3T3) avec une construction T7-CD4 et une banque d'ADNc humains, provenant de cellules Hela, également clonée en aval du promoteur T7. Après infection par le virus vaccine recombinant, les cellules vont synthétiser de grandes quantités de protéines humaines en quelques heures. La présence de T7pol dans les cellules murines permet de détecter, de façon extrêmement sensible, leur éventuelle fusion avec des cellules $\mathrm{Env}^{+}$, préalablement transfectées avec un plasmide T7-lacZ. En effet, leur fusion met en contact T7-lacZ avec T7pol, entraînant une production massive de $\beta$-galactosidase, facilement visualisée par coloration bleue avec le substrat X-gal. Ces événements de complémentation (syncytiums bleus) ont été observés en transfectant la banque entière. Des fractions de moindre complexité ont été testées, et certaines se sont montrées capables de complémenter les cellules murines, avec une efficacité accrue. En répétant cette démarche, un ADNc a finalement été isolé, dont l'expression dans des cellules nonhumaines $\mathrm{CD} 4^{+}$permet la fusion avec des cellules Env ${ }^{+}$et l'infection par les isolats adaptés de VIH-1. Ces expériences démontrent sans ambiguïté qu'il s'agit bien d'un cofacteur d'entrée de VIH-1.

Feng et al. l'ont baptisé fusin, une décision qui peut se discuter, car cette molécule n'est pas à proprement parler nouvelle. Il s'agit d'un membre de la famille des récepteurs à sept domaines transmembranaires ligand connu et donc dit "orphelin " [11], mais relativement proche des récepteurs de l'interleukine 8 (IL-8). Cette parenté avait d'ailleurs permis le clonage de ce récepteur orphelin par plusieurs groupes en 1993-1994, et celui de l'homologue bovin, désigné LCR1 pour locus coeruleus receptor, en 1991 [12]. Outre que le nom fusin ignore ces travaux antérieurs, il met en avant l'entrée de VIH, qui n'est évidemment pas la fonction première de cette molécule.

La parenté de fusin/LCR1 avec les récepteurs de l'IL-8 a permis l'identification d'un facteur de fusion utilisé par les VIH-1 primaires. L'Il-8 est une cytokine chimiotactique, ou chimiokine, participant au recrutement de polynucléaires neutrophiles sur le site de la réponse inflammatoire (pour revue voir [13]). C'est une chimiokine de type $\alpha$, ou C-X-C (les deux cystéines de la partie NH2 terminale sont non adjacentes). Il existe également des chimiokines de type $\beta$, ou C-C (cystéines adjacentes), moins spécifiques car elles attirent plusieurs types de cellules (macrophages, éosinophiles etc.)*. Le groupe de P. Lusso a récemment montré que trois $\beta$-chimiokines, RANTES, MIP- $1 \alpha$, et MIP-1 $\beta$ ont un effet antiviral sur VIH-1, et en particulier sur les souches primaires [14] $\left(\mathrm{m} / \mathrm{s} \mathrm{n}^{\circ} 3\right.$, vol. 12, p. 423). Il était donc naturel de tester le rôle de leurs récepteurs comme facteurs de fusion, et les six types connus de récepteurs des $\beta$ - ou CC-chimiokines ont donc été exprimés dans différentes lignées cellulaires $\mathrm{CD}^{+}$, humaines ou non. Seul CC-CKR5, en présence de $\mathrm{CD} 4$, s'est révélé permettre l'entrée des VIH-1 primaires [2-4]. L'entrée est bloquée par RANTES et MIP-1 $1 \beta$.

Les principaux phénotypes d'entrée de VIH-1 seraient donc expliqués par l'utilisation, soit de fusin/LCR1 (virus adaptés), soit de CC-CKR5 (virus primaires) comme facteur de fusion. On ne peut toutefois exclure

\footnotetext{
* La famille des cytokines apparentées à l'IL-8 est divisée en deux classes sur la base de la présence de deux résidus cystéine conservés, soit ajacents $(C C)$, soit séparés par un acide aminé quelconque $(C X C)$.
}

que d'autres molécules soient utilisées, en particulier pour l'infection des macrophages, car les $\beta$-chimiokines n'ont pas d'effet antiviral pour ces cellules [4]. La situation semble également plus complexe pour VIH-2, les mêmes isolats pouvant utiliser fusin/LCR1, mais aussi infecter des lignées ne synthétisant pas cette molécule, et comme telles réfractaires à VIH-1 (nos observations).

La découverte des facteurs de fusion est une avancée considérable pour la virologie fondamentale. Notre vision de l'entrée de VIH et de la fusion membranaire risque d'évoluer considérablement une fois que seront définis le mécanisme de l'interaction de l'enveloppe virale avec les facteurs de fusion, et ses conséquences éventuelles pour la cellule, via le couplage aux protéines G. Ces travaux peuvent aussi avoir des retombées pratiques. On sait depuis quelques années que certains sujets sont apparemment résistants à l'infection par VIH-1, et que cette résistance s'observe aussi ex vivo quand leurs lymphocytes sont exposés à des isolats VIH-1 primaires (mais non à des isolats adaptés). Dragic et al. suggèrent que ces individus pourraient avoir un allèle de CCCKR5 non fonctionnel [4]. Si ce point est confirmé, il devrait être en théorie possible de bloquer ce récepteur, et donc d'exercer une action antivirale, sans affecter la réponse aux $\beta$-chimiokines. On restera cependant prudent sur ces perspectives thérapeutiques, surtout si les chimiokines n'ont pas d'effet antiviral pour les macrophages, cibles principales du virus au début de l'infection. De même, la découverte des facteurs de fusion ouvre théoriquement la possibilité d'obtenir des souris transgéniques infectables. Mais la faible expression de VIH-1 dans les cellules murines risque d'empêcher d'établir une infection productive chez ces animaux, même si le bloc d'entrée est surmonté. L'expression de VIH-1 semble relativement plus efficace dans les cellules de lapin, et cet animal pourrait être un meilleur modèle transgénique.
M.A. 
1. Feng Y, Broder CC, Kennedy PE, Berger EA. HIV-1 entry cofactor: functional cDNA cloning of a seven-transmembrane G-protein coupled receptor. Science 1996; 272: 872-7.

2. Alkhatib G, Combadiere C, Broder CC, Feng Y, Kennedy PE, Murphy PM, Berger EA. CC CKR5: a RANTES, MIP- $1 \alpha$, MIP-1 $\beta$ receptor as a fusion cofactor for macrophage-trophic HIV-1. Science $1996 ; 272: 1955-8$.

3. Deng H, Liu R, Elmeier W, Choe S, Unutmaz D, Burkhart M, Di Marzio P, Marmon S, Sutton RE, Hill CM, Davis CB, Peiper SC, Schall TJ, Littman DR, Landau NR. Identification of a major co-receptor for primary isolates of HIV-1. Nature 1996; 381: 661-6. 4. Dragic T, Litwin V, Allaway G, Martin SR, Huang Y, Nagashima KA, Cayanan C, Maddon PJ, Koup RA, Moore JP, Paxton WA. HIV-1 entry into $\mathrm{CD}^{+}$cells is mediated by the chemokine receptor CC-CKR5. Nature 1996 ; 381 : 667-73.

5. Maddon PJ, Dalgleish AG, McDougal JS, Clapham PR, Weiss RA, Axel R. The T4 gene encodes the AIDS virus receptor and is expressed in the immune system and the brain. Cell $1986 ; 47$ : 333-48.

6. Lazaro I, Naniche D, Signoret N, Bernard A-M, Marguet D, Klatzmann D, Dragic T, Alizon M, Sattentau Q. Factors involved in entry of the human immunodeficiency virus type 1 into permissive cells : lack of evidence of a role for CD26. J. Virol $1994 ; 68: 6535-46$.

7. Tersmette M, Van Dongen JJM, Clapham PR, DeGoede REY, Wolvers-Terrero ILM, Geurts van Kessel A, Huisman JG, Weiss RA \& Miedema F. Human immunodeficiency virus infection studied in CD4-expressing human-murine T-cell hybrids. Virology 1989 ; 168 : 267-73.

8. Dragic T, Charneau P, Clavel F, Alizon M. Complementation of murine cells for human immunodeficiency virus envelope/CD4-mediated fusion in human-murine heterokaryons. J Virol 1992; 66 : 4794-802.

9. Dragic T, Picard L, Alizon M. Proteinase-resistant factors in human erythrocyte membranes mediate CD4-dependent fusion with cells expressing human immunodeficiency virus type 1 envelope proteins. J Virol 1995 ; 69 : 1013-8.

10. Clapham PR, Blanc D, Weiss RA. Specific cell surface requirements for the infection of CD4-positive cells by human immunodeficiency virus types 1 and 2 and by simian immunodeficiency virus. Virology $1991 ; 181$ : 703-15.

11. Parmentier M, Libert F, Vassart G. La famille des récepteurs couplés aux protéines $\mathrm{G}$ et ses orphelins. médecine/sciences $1995 ; 11: 222-31$.

12. Rimland J, Xin W, Sweetnam P, Saijoh K, Nestler EJ,Duman RS. Sequence and expression of a neuropeptide Y receptor cDNA. Mol Pharmacol $1991 ; 40: 869-75$.

13. Minty AJ. Une nouvelle famille de cytokines inflammatoires. médecine/sciences $1991 ; 7$ : 57888.

14. Lusso P, DiMarzo Veronese F, Ensoli B, Franchini G, Jemma C, DeRocco SE, Kalyanaraman VS, Gallo RC. Expanded HIV-1 cellular tropism by phenotypic mixing with murine endogenous retroviruses. Science $1990 ; 247$ : 848-51.

\section{FONDATION FYSSEN

\author{
194, RUE DE RIVOLI - 75001 PARIS, FRANCE \\ TÉL. : 33 (1) 42.97.53.16 \\ FAX : 33 (1) 42.60 .17 .95
}

La FONDATION FYSSEN a pour objectif général «de promouvoir sous toutes ses formes l'analyse scientifique des mécanimses logiques du comportement animal et humain ainsi que leur développement ontogénétique et phylogénétique».

\section{BOURSES D’ÉTUDES POST-DOCTORALES}

La FONDATION FYSSEN attribuera un certain nombre de BOURSES D'ÉTUDES.

Ces bourses doivent permettre la formation et le soutien de chercheurs de niveau post-doctora travaillant dans des domaines de recherche qui répondent aux objectifs de la Fondation tels que l'éthologie, la paléontologie, l'archéologie, l'anthropologie, la psychologie, l'épistémologie, la logique et les sciences du système nerveux.

La Fondation souhaiterait soutenir plus particulièrement les recherches dans les domaines tels que : ÉTHOLOGIE ET PSYCHOLOGIE : La nature et le développement des processus cognitifs chez l'homme et chez les animaux. Le déterminisme des comportements au cours de l'ontogenèse et leur évolution à travers la phylogenèse.

NEUROBIOLOGIE : Les études portant sur les bases neurobiologiques des processus cognitifs et de leur développement embryonnaire et post-natal ainsi que les mécanismes élémentaires qu'ils engagent. ANTHROPOLOGIE-ETHNOLOGIE : L'étude :

a) des systèmes de représentations des environnements naturels et des cultures. Analyse des principes de construction et des mécanismes de transmission de ces systèmes en mettant en évidence leurs aspects cognitifs ;

b) des systèmes techniques développés dans les diverses formes d'organisation sociale et analysés sous tous leurs aspects (savoirs, savoir-faire, mécanismes de transmission).

PALÉONTOLOGIE HUMAINE : L'origine et l'évolution du cerveau humain et de ses productions.

Ces bourses d'un montant maximum de $120000 \mathrm{~F}$ annuel seront réservées à des chercheurs français désirant se rendre dans des laboratoires étrangers et à des chercheurs étrangers venant travailler dans des laboratoires français. Elles s'adressent aux jeunes chercheurs (moins de 35 ans) et sont normalement d'une durée maximale d'un an ; elles peuvent éventuellement être renouvelées.

Les demandes de bourses doivent être établies suivant un formulaire à demander à la Fondation de Septembre à Février et qui comportera notamment :

- le curriculum vitae

- la liste des publications du candidat,

- les lettres de deux personnalités scientifiques le connaissant et susceptibles de porter une appréciation sur ses travaux,

- l'acceptation écrite du laboratoire d'accueil.

Les dossiers complets doivent être adressés en 15 exemplaires au Secrétariat de la Fondation, 194, rue de Rivoli, 75001 PARIS.

BOURSES : Date limite impérative de réception des dossiers : le 31 mars 1996.

Seules seront prises en considération les demandes de bourses qui entrent explicitement dans les objectifs de la Fondation.

\section{PRIX INTERNATIONAL}

UN PRIX INTERNATIONAL de $200.000 \mathrm{~F}$ sera attribué à un chercheur qui se sera distingué par une activité de recherche fondamentale qui correspond, directement ou indirectement, à l'objectif de la Fondation et qui concerne des disciplines telles que l'éthologie, la paléontologie, l'archéologie, l'anthropologie, la psychologie, l'épistémologie, la logique et les sciences du système nerveux. II a été décerné à MM. les Professeurs A. LEROIGOURHAN (1980), W.H. THORPE (1981), V.B. MOUNTCASTLE (1982), H.C. CONKLIN (1983), R.W. BROWN (1984), P. BUSER (1985), D. PILBEAM (1986), D. PREMACK (1987), J.C. GARDIN (1988), Mme P.S. GOLDMAN-RAKIC (1989), J. GOODY (1990), G.A. MILLER (1991), P. RAKIC (1992) et L.L. CAVALLI-SFORZA (1993), L. GLEITMAN (1994).

Discipline considérée pour le Prix 1996 :

\section{TECHNIQUES ET SYMBOLES AU COURS DE L'ÉVOLUTION HUMAINE}

Les propositions de candidature doivent comporter :

- le curriculum vitae,

- la liste des publications du candidat,

- un résumé (quatre pages maximum) du travail de recherche qui justifie l'attribution du Prix.

On ne peut se porter directement candidat.

Les candidatures au PRIX de la FONDATION FYSSEN doivent être adressées en 15 exemplaires au Secrétariat de la Fondation, 194, rue de Rivoli, 75001 PARIS.

PRIX : Date limite des propositions de candidature : le $1^{\text {er }}$ octobre 1996. 